\title{
Application of indicator in factor analysis on transport energy consumption
}

\author{
JIANG Jian \\ School of Traffic and Transportation, Beijing Jiaotong University, Beijing 100044, China \\ Email: jjiang@bjtu.edu.cn
}

\begin{abstract}
Keywords: sustainability; transport; indicator; energy consumption
Abstract. As the energy issue is getting more and more serious with the economy development of the world, the research on the issue of transport and logistics sustainability is becoming more and more popular for researchers all around. For one respect, the restriction of energy consumption in transport sector for logistics is crucially important to a certain extent. There are many ways for analyzing the factors of the superfluous energy consumption in transport to give reasonable interpretation for the incentives of it. In recent years, factor analysis relating method such as decomposition analysis and data envelopment analysis are used in literatures in order to scratch the principal factor that leads to the consumption. However, it remains a problem that why the very factors are selected for the analysis instead of others, and there is no convincing evidence given by the analyzers. In this paper, we decide to use the method of indicator analysis rather than generally used factor analysis to make the analysis afterwards more reasonable and give application of the method. Indicator analysis considers the possible factor to be candidate indicator and evaluate them in advance based on the criteria of indicator selection. The criteria are developed for the application in the transport domain and classified into three categories which named representation, operation and policy application. The scoring criteria for evaluation are also given in detail in order to get impartial result.
\end{abstract}

\section{Introduction}

Sustainable development is considered to be a permanent topic around the world in recent years. The basic sprit of sustainability is to meet human being's needs as well as preserving the resources and environment of the world, so that the needs of human can be met not only at the time being, but also in the future for next generations. Sustainable is the systematically combination of environment, economic and social. Based on this spirit, we have to take more consideration on sustainability as well as economic development. Transport activity effects the environment, economic and social very much, therefore it is very important to trace the activity of transport for the research of activity. In this paper, we focus on the domain of the energy consumption in transport factor. The transport sector accounts for about $20 \%$ in the total all over the world, and the absolute amount is increasing continuously, which is rather a crucial part.

There are many ways to analyze the factors lead to the change of the consumption of the energy consumption for further policy suggestion. The method mostly used is to analyze the factors which lead to the change of the total amount of consumption. Thanks to the method of factor analysis, we can find out the importance of each factor and can give corresponding suggestions on improvement. For instance, we can calculate the proportion of each factor introduces by the method of decomposition analysis [1]. However, the problem is that the selection on the choosing of factors does not have certain criteria at all. It will lead to totally different result of analyzing based on different selections of factors. Corresponding references are listed for the issue in detail. For, further detail information, please refer to references 2-6.

In this paper, we introduce the concept of indicator for the analysis of the energy consumption in transport sector rather than common factor analysis and scribe detail criteria for the application of it.

\section{Indicator analysis}

Indicator is defined as a substance used to show visually the condition of solution with respect to the presence of a particular material by the Webster's dictionary. In the field of abstraction, it would give 
out invisible implications with the function as a maker or sentinel, indicating the presence or absence, a measurement tool, indicating variations along important dimensions of phenomenon, a decision support tool allowing taking certain action, or the combination of the above [7].

As indicators have the function to indicate the phenomenon of transport production, on the issue of energy consumption, we should analyze it in order to grasp the nature of the change of energy consumption for policy suggestions. The remaining of the issue relies on the way for the selection from factors into indicators. According to the reference the criteria of indicator selecting can be defined as follows as most researchers agreed. We also believe that the three categories with ten criteria are the most reasonable ones.

The ten criterion of three categories are listed below[7]:

1. Validity. A valid indicator must actually measure the issue or factor it is supposed to measure A valid indicator must be based on a conceptual model that justifies how the indicator and the issue are causally connected.

2. Reliability. A reliable indicator must consistently give the same value if its measurement is repeated in the same way on the same population and at approximately the same timeIn other words, the achievement of information for the phenomenon should be repeatable.

3. Sensitivity. A sensitive indicator must be able to reveal important changes in the factor of interest. Indicators should generally react clearly and promptly to significant changes in the phenomenon being investigated.

4. Measurability. A measurable indicator should be straightforward and relatively inexpensive to measure. It is important that an indicator is measurable and calculable using simple tools and simple data that are easily achievable and at a raw level.

5. Data availability. Indicators with data availability are based on input data that are readily available or can be made available at reasonable cost and time. The data should be accurate, comparable over time, complete with historical information, and cover a sufficient geographic area.

6. Ethical concerns. An indicator must comply with fundamental human rights and require only data consistent with the morals, beliefs, and values of the population. This is to ensure that no human right is violated during the acquisition of the information required to operate the indicator.

7. Transparency. A transparent indicator is one that intended users can understand and reproduce. This means that an indicator must describe in an understandable way how the indicator is constructed.

8. Interpretability. An interpretable indicator allows for an intuitive and unambiguous reading. It should be easy to understand whether an indicator is a positive variable or a negative variable.

9. Target relevance. A target-relevant indicator must measure performance with regard to articulated goals, objectives, targets, or thresholds. This criterion means that the selection of the indicator should meet the needs of the objectives and goals for the phenomenon analysis.

10. Actionability. An actionable indicator measures factors that can be changed or influenced directly by management or policy action. Actionability signifies the role of indicators as tools that support management and decision making.

However, the literature assumes that all the ten criteria are of the same importance as they share the same value, at the same time, the evaluation of each criteria are almost decided subjectively, and the variance of the scores cannot be controlled, too. In fact, these ten criteria may be not of the same importance; therefore, the result of the scoring would not be so precise for us to select. In order to solve this problem for the practical analysis of the issue, we analyze the criteria one by one for the very issue on the consumption of energy in transport sector.

First, we lay out the candidate indicators that may be the one affects the energy consumption of transport crucially according to Peter W.G Newman, Jeffery R. Kenworthy, 1989[8]. Note that some factors are applied on the macro analysis all over a wide region while some factors are only applied on cities. It is important to tell them apart on the choice of factors into candidate indicator for practical 
analysis case. Moreover, cluster analysis maybe necessary for confirming the same shape of analysis objects.

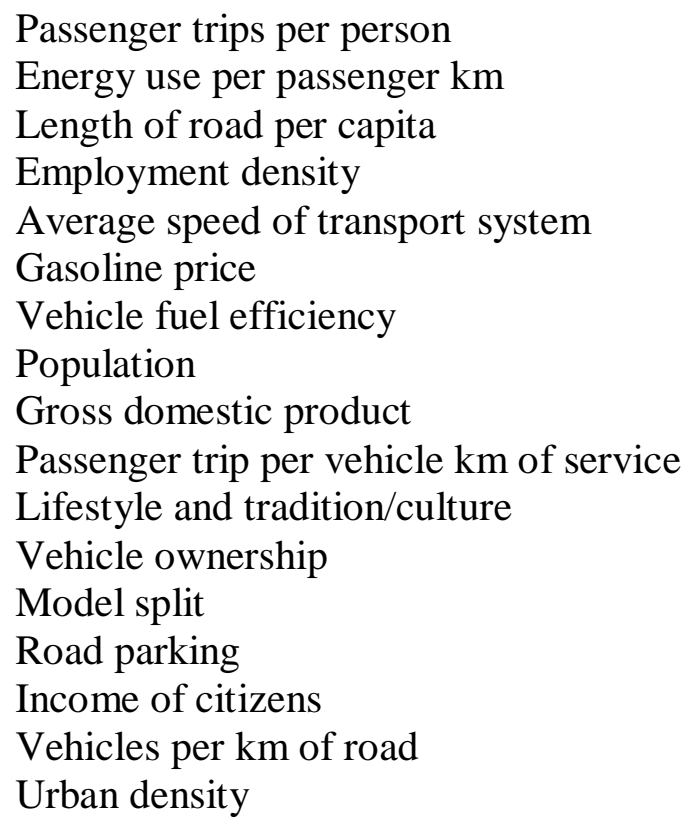

This issue has little connection of ethical concerns, so we ignore this criterion for scoring. Data availability is crucial important because it is necessary to get every data for the analysis based on quantitative calculation. The other criteria can be decided subjectively as long as the decision is stable. We mark it from level 1 to 4 to correspond the fitness of each candidate from poor, fair, good and excellent.

From the example factors introduced above, we notice that the factors are not all in the same field as every analysis concerns; therefore, we would like to analyze the fitness of factors into indicators by criteria according to the problem we are facing rather than putting all the probable factors into consideration.

Based on these calculations, we can deduce the evaluation of each candidate easily and lead to further results.

\section{Applications and conclusion}

In the literature 9-10, DEA method was used for the analysis on the environmental efficiency. Population density, road length per capita, private car ownership per capita and public transport serve length per capita are selected as input variables for analysis without further explanation for the reason. And the result will be totally different if other variables are selected. Although the choice in the paper sound reasonable in certain degree and the result is very useful and robust, had the method of indicator analysis been introduced in, the analysis result would be more convincing.

This is an example for the application of the indicator analysis when it is used in DEA method. Here we want to solve the problem for the analysis of environmental efficiency among cities to see which one does better on transport sustainability than others by DEA method. The output variable is decided for the transport energy consumption, what we want to do with the help of the indicator analysis is to pick up the input variables for the calculation. The result is shown as Table.1. Based on this result, the former 4 candidates are elected for the input variable for further analysis. And what's more, the calculation for the environmental efficiency among cities will become more reasonable. 
Table 1. The application of indicator selection on DEA

\begin{tabular}{|c|c|c|c|c|c|c|c|}
\hline Category & Criteria & $\begin{array}{c}\text { intensity of } \\
\text { population }\end{array}$ & $\begin{array}{c}\text { number } \\
\text { of cars }\end{array}$ & $\begin{array}{c}\text { length } \\
\text { of road }\end{array}$ & $\begin{array}{c}\text { intensity } \\
\text { of road }\end{array}$ & $\begin{array}{c}\text { average } \\
\text { speed of } \\
\text { vehicles }\end{array}$ & $\cdots$ \\
\hline \multirow{3}{*}{ Representation } & Validity & 2 & 4 & 3 & 3 & 2 & $\ldots$ \\
\cline { 2 - 8 } & Reliability & 4 & 4 & 4 & 4 & 4 & $\ldots$ \\
\cline { 2 - 8 } & Sensitivity & 4 & 4 & 4 & 4 & 3 & $\ldots$ \\
\hline Operation & Measurability & 4 & 4 & 4 & 4 & 2 & $\ldots$ \\
\hline \multirow{3}{*}{ Application } & Transparency & 4 & 3 & 4 & 4 & 2 & $\ldots$ \\
\cline { 2 - 8 } & Interpretability & 3 & 3 & 2 & 4 & 2 & $\ldots$ \\
\cline { 2 - 8 } & Target & 2 & 4 & 3 & 3 & 3 & $\ldots$ \\
\cline { 2 - 8 } & relevance & & 4 & 4 & 4 & 3 & $\ldots$ \\
\cline { 2 - 9 } & Action ability & 3 & 30 & 28 & 30 & 21 & $\ldots$ \\
\hline
\end{tabular}

\section{Acknowledgements}

This work was financially supported by AQSIQ quality inspection of public welfare industry research projects (201510210-03).

\section{References}

[1] Ang,B.W.,Zhang,F.Q.,2000. A survey of index decomposition analysis in energy and environmental studies. Energy25, pp.1149-1176.

[2] C.Bockstaller and P. Girardin, 2003. How to validate environmental indicators. Agricultural Systems 76. pp.639-653.

[3] David Niemeijer and Rudolf S. de Groot, 2008. A conceptual framework for selecting environmental indicator sets. Ecological Indicators 8. pp.14-25

[4] Peter Hardi and Juanita Ama DeSouza-Huletey, 2000. Issues in analyzing data and indicators for sustainable development. Ecological Modelling 130. pp.59-65

[5] Todd Litman, 2011. Developing Indicators for Comprehensive and Sustainable Transport Planning. Transport Policy.

[6] United States Environmental Protection Agency, 1996. Indicators of the Environmental Impacts of Transportation-Highway, Rail, Aviation, and Maritime Transport.

[7] Jourmard and Gudmundsson 2010. Indicators of environmental sustainability in transport-An interdisciplinary approach to methods. Recherches.

[8] Peter W.G Newman, Jeffery R. Kenworthy, 1989. Cities and automobile dependence: A sourcebook

[9] Yoshino D., Fujiwara A. Potential Reduction of CO2 Emissions in Transport Sector and Its Countermeasures Based on DEA, Proceedings of Infrastructure Planning Vol.41 CD-ROM,2010

[10] Yoshino,D.,Fujiwara,A.,and Zhang,J.2010. Environmental efficiency model based on data envelopment analysis and its application to environmentally sustainable transport policies, the 89th Annual meeting of the transportation research board CD-ROM 